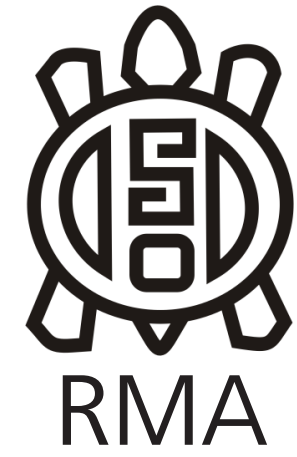

Dossier

\title{
Aplicación de la técnica de cromatografía gaseosa para el análisis de lípidos sobre artefactos líticos provenientes del Macizo del Deseado: primeros resultados
}

\author{
Gas chromatograhy applied to the analysis of lithic artifacts from the \\ Deseado Massif: first results \\ Pablo E. Bianchi* y Valeria P. Careaga**
}

*Instituto Multidisciplinario de Historia y Ciencias Humanas, CONICET y Agencia Nacional de Promoción Científica y Técnica, Argentina. E-mail: bianchipabloem@gmail.com ${ }^{*}$ CONICET, Unidad de Microanálisis y Métodos Físicos en Química Orgánica. E-mail: pcareaga@qo.fcen.uba.ar

\begin{abstract}
Resumen
Este trabajo constituye una primera aproximación al estudio, desde una perspectiva arqueométrica, de las estrategias de aprovechamiento de recursos naturales por parte de los grupos cazadores recolectores que habitaron el extremo sur del Macizo del Deseado (provincia de Santa Cruz) durante el Holoceno tardío. A tal fin, se planteó el estudio de un conjunto de artefactos líticos recuperados en el sitio La Gruta 3 mediante la aplicación de la técnica de cromatografía gaseosa. Por otro lado, la investigación implicó el desarrollo y realización de una experimentación controlada, a fin de conocer cómo reaccionan los artefactos líticos ante el contacto con elementos que poseen lípidos en su composición. Esto involucró la confección de piezas capaces de ser utilizadas para el procesamiento de recursos faunísticos, posteriormente analizadas utilizando las técnicas mencionadas. Los resultados obtenidos dieron cuenta de la capacidad del material lítico para preservar sustancias -lípidos- a partir del contacto con recursos de diverso origen. En este sentido, los análisis sobre artefactos arqueológicos permitieron recuperar ácidos grasos plausibles de ser relacionados con especies animales locales, aunque no deben descartarse las especies vegetales. La conservación de estos componentes a lo largo del tiempo, a pesar de estar sujetos a diferentes procesos que puedan alterar su integridad, alienta la profundización en el estudio de artefactos arqueológicos mediante la utilización de técnicas de caracterización química. Este trabajo permite reconocer el valor de los enfoques arqueométricos para la resolución de problemas arqueológicos particulares, atravesados por dificultades respecto de la conservación de la evidencia o por el tipo de interrogante planteado.
\end{abstract}

Palabras clave: Macizo del Deseado; Lítico; Lípidos; Cromatografía gaseosa; Ácidos grasos.

\begin{abstract}
This study constitutes a first step to the understanding, from an archaeometric perspective, of the resource procurement strategies used by the human hunter-gatherers in the Southern end of the Deseado Massif (Santa Cruz province) during the Late Holocene. Archaeological samples from La Gruta 3 rockshelter were analyzed using gas chromatography. An experimental activity was carried on, in order to have a control sample that allows us to account how the local raw material behaves in contact with fatty substances. The results have shown the capacity of lithic materials to preserve them and, in the case of archaeological artifacts, to connect them with animal resources, though vegetables can't be discarded. The preservation of these components in time, in spite of being subjected to processes that can alter their integrity, encourages the analysis of archaeological evidence using chemical characterization techniques. This study recognizes the importance of archaeometric approaches for the resolution of specific archaeological queries intertwined with sample conservation problems or by the kind of question asked.
\end{abstract}

Key words: Deseado Massif; Lithic; Lipids; Gas chromatography; fatty acids.

\section{Antecedentes}

Las primeras ocupaciones humanas en Patagonia argentina se identifican arqueológicamente durante la transición Pleistoceno final - Holoceno temprano, un período caracterizado por variaciones medioambientales -especialmente cambios en la humedad y temperatura- en el marco de las cuales se desarrolló la dispersión inicial y colonización del espacio por parte de grupos cazadores recolectores (Borrero et al. 1998; Miotti y Salemme 2002; Mancini et al. 2013). En el sur del Macizo del Deseado (provincia de Santa Cruz) se identificaron ocupaciones tempranas (entre 12.799 y 12.049 Cal AP) en un pequeño reparo rocoso en cercanías de una laguna: el sitio La Gruta 1 (LG1). Estas se continúan, en forma discontinua, durante el Holoceno temprano y medio (Franco et al. 
2013), habiéndose ocupado también otras cuevas (La Martita Cueva 4 y El Verano Cueva 1) entre 10.294 y 7.760 Cal AP (Aguerre 2003; Durán et al. 2003; Brook et al. 2015). En momentos tardíos se identificaron nuevas ocupaciones en los sitios mencionados. La subsistencia humana se basó principalmente en el consumo de guanaco (Lama guanicoe), aunque otras especies como choique (Pterocnemia pennata), zorro gris (Pseudalopex griseus), zorro colorado (Pseudalopex culpaeus) y tucu tucu (Ctenomys colburni) fueron ampliamente explotadas. Sin embargo, existe escasa información acerca de la utilización de otros recursos, especialmente aquellos de origen vegetal.

El objetivo del presente trabajo es, a partir de la información proveniente del análisis de ácidos grasos, realizar una primera aproximación al estudio de los diversos recursos aprovechables en área bajo estudio, a fin de complementar la información disponible hasta el momento.

\section{Materiales y Métodos}

Los artefactos arqueológicos ( $\mathrm{N}=4$ ) y las muestras de sedimento $(\mathrm{N}=2)$ seleccionadas provienen de los depósitos asignados al Holoceno tardío del sitio La Gruta 3 (LG3), datados entre 539 y 156 Cal AP (Brook et al. 2015). Se trata de un abrigo rocoso perteneciente a la formación Monte León ubicado en un sector caracterizado por abundantes afloramientos de areniscas coquinoideas gruesas (Panza y Marín 1998: 38), muy próximo a una laguna cuya disponibilidad de agua es escasa, aunque han sido registrados eventos de crecimiento de su caudal. Los artefactos seleccionados -tanto arqueológicos como experimentales- fueron analizados en forma previa a la realización de los estudios arqueométricos siguiendo los lineamientos propuestos por Aschero (1975 y 1983), a fin de caracterizar tecno-morfológicamente la muestra seleccionada.

\section{Ácidos grasos}

Los lípidos son moléculas orgánicas hidrofóbicas formadas por átomos de $\mathrm{H}, \mathrm{C}$ y O. De acuerdo con su estructura, pueden clasificarse en insaponificables y saponificables. Estos últimos contienen ácidos grasos, que se diferencian entre sí por la longitud de la cadena hidrocarbonada, presencia, número y posición de los dobles enlaces. A su vez, pueden ser divididos en saturados (contienen enlaces simples) o insaturados (poseen uno o más enlaces dobles). La cromatografía gaseosa, en tanto, es una técnica que permite separar los componentes volátiles de una mezcla, la cual puede emplearse para analizar los extractos lipídicos recuperados de soportes de diversa composición, producto del contacto con diversos tipos de recursos. Su aplicación a estudios arqueológicos se profundizó hacia la década de 1990, y se orientó al estudio de aspectos relacionados con la dieta de las poblaciones, así como con las diversas formas de preparación y consumo de alimentos (Evershed et al. 1990; Malainey et al 1999 a y b, Evershed 2008). Gran parte de los trabajos realizados se centraron en el análisis de fragmentos de cerámica, debido a que su matriz porosa permite la preservación de lípidos (entre otros, Cordero y March 2013; Lantos 2013; Stoessel et al. 2015; Frére et al. 2016; Chaile et al. 2018). Su aplicación sobre material lítico es escasa (Buonasera 2005 y 2007; Babot et al. 2007; Mazzia 2010; Gómez Otero et al. 2014; Mazzia et al. 2016).

A partir del interés en evaluar la aplicación de la técnica sobre los lípidos en este tipo de material arqueológico, surgió la necesidad de poseer una muestra artefactual de control que permitiera evaluar el comportamiento de la materia prima local ante el contacto con material graso. Se diseñó entonces una experimentación que consistió en el procesamiento de recursos faunísticos actuales utilizando artefactos confeccionados en materias primas propias del área bajo estudio. Se seleccionaron dos piezas cárnicas pertenecientes a mamíferos actuales: vaca (Bos primigenius taurus) y cerdo (Sus scrofa domesticus). Ambas cumplieron una condición importante para la realización de la experimentación: poseer cuero, grasa, carne y hueso. Paralelamente, se procedió a la talla experimental de instrumentos líticos a partir de materias primas provenientes del área bajo análisis, los cuales fueron utilizados para procesar los mencionados cortes cárnicos. Se recolectaron en campo un conjunto de guijarros de sílice y calcedonia, dos tipos de roca con una abundancia y disponibilidad espacial conocida, tanto natural como arqueológicamente. Los guijarros seleccionados no poseían evidencias de utilización y debían poseer un tamaño adecuado para la

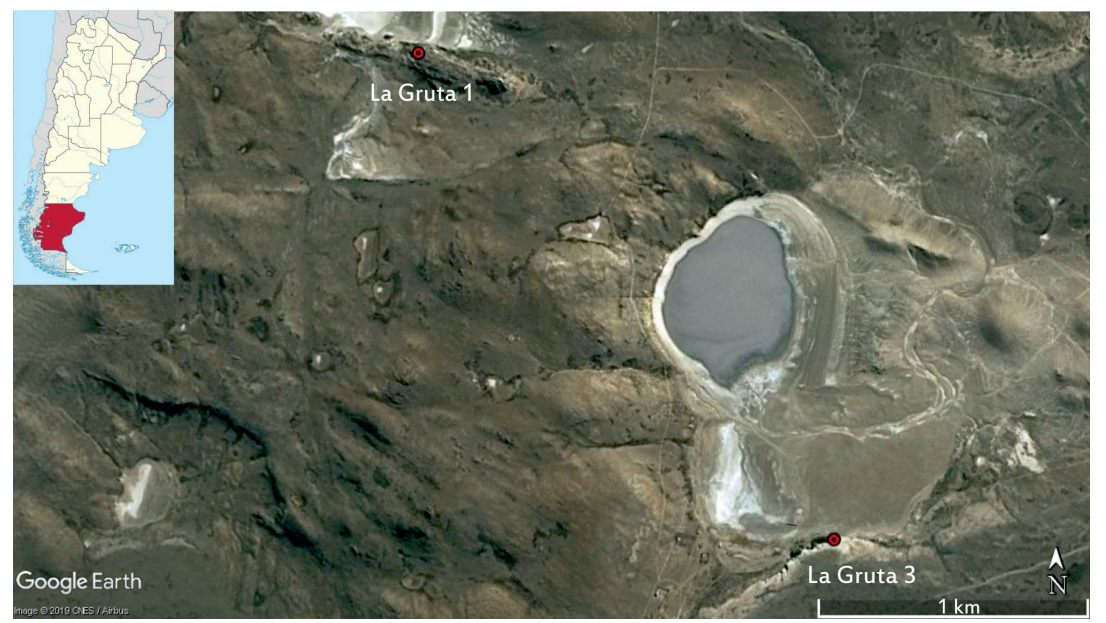

Figura 1. Ubicación de los sitios La Gruta 1 y 3 .

Figure 1. Location of archaeological sites La Gruta 1 and 3. 
extracción de lascas. Al tratarse de materias primas con diferentes características composicionales, la actividad de procesamiento permitió obtener información acerca del comportamiento de cada una respecto de la preservación de sustancias grasas. En el caso del cerdo, se procesó un jamón de cerdo (incluyendo fémur y tibia), cuyo peso inicial fue de $7,730 \mathrm{~kg}$. La duración total de la actividad de procesamiento fue de 2:30 horas, período en el cual fueron separados los diferentes componentes (cuero, grasa, carne y hueso). Asimismo, fueron utilizados 9 artefactos, entre los que se cuentan raederas y lascas con filo natural. En el caso de la vaca, se trató de un morcillo delantero (con fragmento proximal de radio cúbito), cuyo peso inicial fue de 2,660 kg. El procesamiento de la pieza se extendió durante 1 hora 30 min, período en el cual fueron separados los diferentes componentes (cuero, grasa, hueso). Fueron utilizados 9 artefactos, entre los que se cuentan raederas y lascas con filo natural. El promedio de contacto de cada artefacto con el recurso fue de 10 minutos.

Los artefactos experimentales estuvieron en contacto con las piezas cárnicas por períodos de tiempo diferentes y fueron utilizados en repetidas ocasiones, de acuerdo con las elecciones realizadas por el operador en las diferentes etapas de procesamiento. El promedio de contacto de cada uno con el recurso fue de unos 15 minutos, influyendo factores tales como el tamaño total de las piezas cárnicas, la presencia de cuero, tejido graso y hueso. Una vez finalizada la actividad experimental, se seleccionaron 2 artefactos a fin de obtener los extractos lipídicos para ser analizados, por cromatografía de gases con detector de ionización de llama (CG-FID) y cromatografía gaseosa acoplada a espectrometría de masas (CG-EM): una raedera de sílice con reserva de corteza (cerdo) y una lasca de calcedonia castaño translúcida con filo natural (vaca). En cuanto al conjunto arqueológico, se seleccionaron 4 artefactos para ser sometidos al mismo proceso: un cuchillo fragmentado, una lasca de arista, una lasca angular y un raspador sobre hoja. Paralelamente, se analizaron muestras de los sedimentos correspondientes a los estratos que contenían las muestras arqueológicas seleccionadas, a efectos de evaluar la posibilidad de contaminación por absorción de lípidos provenientes de la matriz que las contenía. En todo momento, los artefactos fueron manipulados con guantes de nitrilo, a fin de evitar contaminación por contacto con sustancias presentes en la piel humana y, posteriormente, conservados en bolsas de polipropileno tipo zipper. Los análisis fueron realizados en el laboratorio perteneciente a la Unidad de Microanálisis y Métodos Físicos Aplicados a Química Orgánica (UMYMFOR-FCEN-UBA).

Las piezas se extrajeron con una mezcla cloroformo/ metanol $(2: 1)$ y se sonicaron durante 15 minutos. Se extrajo 2 veces cada muestra. Los lípidos obtenidos fueron transmetilados con cloruro de acetilo en metanol durante 2 horas a $70^{\circ} \mathrm{C}$ en estufa. Una vez que se enfrió la muestra, se agregó una gota de agua y se extrajo con cloroformo. Los esteres metílicos obtenidos en el paso anterior se secaron bajo atmósfera de nitrógeno y se analizaron por CG-EM y CG-FID, con el propósito de identificar y cuantificar los ácidos grasos, respectivamente.

Para el análisis por CG-FID se utilizó un equipo Focus GC (Thermo Finnigan Corporation), equipado con una columna capilar HP Innowax (100\% polyetileneglicol, 30 m, 0.25 $\mathrm{mm}$ i.d., $0.5 \mu \mathrm{m}$ film thickness) (Agilent Technologies USA). Flujo del gas carrier (nitrógeno): $0.8 \mathrm{~mL} / \mathrm{min}$. Inyección manual en modo splitless; temperatura del inyector: $220^{\circ} \mathrm{C}$. La temperatura del detector fue seteada a $275^{\circ} \mathrm{C}$. Programa de temperatura empleado: $100^{\circ} \mathrm{C}(1 \mathrm{~min}), 15^{\circ} \mathrm{C} /$ min hasta $200^{\circ} \mathrm{C}(1 \mathrm{~min}), 2^{\circ} \mathrm{C} / \mathrm{min}$ hasta $240^{\circ} \mathrm{C}(17 \mathrm{~min})$.

En el caso del análisis por CG-EM se utilizó un equipo Shimadzu GCMS -QP5050A (Kyoto, Japan), equipado con una columna capilar HP Innowax (100\% polyetileneglicol, $30 \mathrm{~m}, 0.25 \mathrm{~mm}$ i.d., $0.25 \mu \mathrm{m}$ film thickness) (Agilent Technologies USA). Flujo del gas carrier (helio): 1,1 mL/min. Inyección manual en modo split; temperatura del inyector: $250^{\circ} \mathrm{C}$. La temperatura del detector fue seteada a $290^{\circ} \mathrm{C}$. Programa de temperatura empleado: $90^{\circ} \mathrm{C}(2 \mathrm{~min}), 15^{\circ} \mathrm{C} / \mathrm{min}$ hasta $200^{\circ} \mathrm{C}(1 \mathrm{~min}), 2^{\circ} \mathrm{C} / \mathrm{min}$ hasta $240^{\circ} \mathrm{C}(15 \mathrm{~min})$.

\section{Resultados}

El trabajo realizado arrojó resultados positivos, detectándose la presencia de lípidos en todas las muestras analizadas. En el caso específico de los artefactos experimentales, el contacto con las piezas cárnicas -aún en un período de exposición relativamente acotadopermitió comprobar la preservación de estas sustancias en la matriz porosa de las rocas. Por otra parte, tanto los artefactos arqueológicos como las muestras de sedimento analizadas por cromatografía gaseosa evidenciaron la presencia de lípidos compuestos por distintos ácidos

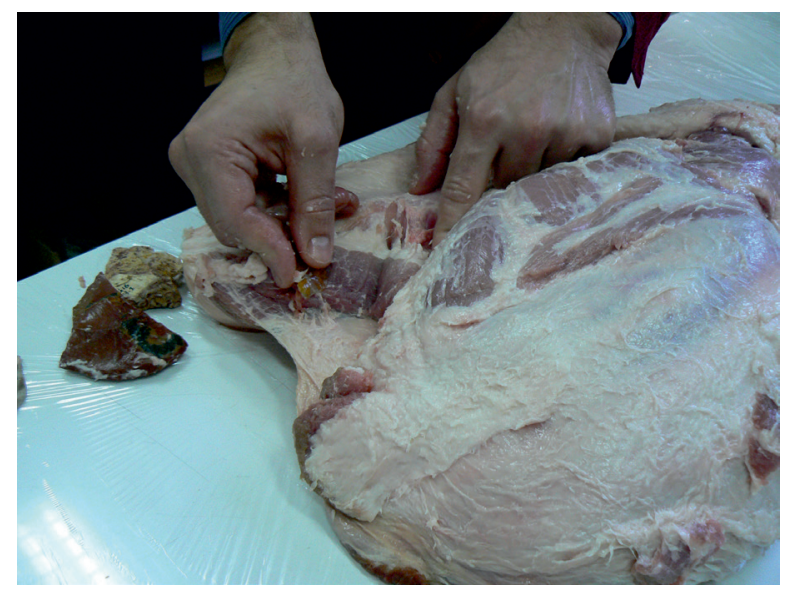

Figura 2. Procesamiento de pieza cárnica (Sus scrofa domesticus).

Figure 2. Processing of a part of a carcass (Sus scrofa domesticus). 
grasos y en concentraciones diversas. En la Tabla 1 puede observarse la abundancia relativa de ésteres metílicos de ácidos grasos (FAME) obtenidos por CG-FID. Los resultados obtenidos permitieron caracterizar el perfil lipídico de todos los artefactos, a excepción del caso LG3 299 donde solo identificaron trazas. El ácido palmítico o hexadecanoico (C16:0) fue el componente detectado en mayor proporción en las muestras seleccionadas, mientras que los porcentajes de ácido octadecanoico obtenidos en las muestras LG3 253 (18,01\%), LG3 298 $(6,29 \%)$ y LG3 $313(20,15 \%)$ y de ácido tetradecanoico son destacables. También es importante resaltar la presencia de ácidos de cadena impar como el ácido pentanodioco (C15:0) -observado tanto en forma lineal como ramificado- y el heptadecanoico (C17:0). Estos compuestos están reportados en las grasas de animales rumiantes, debido a la actividad bacteriana desarrollada en el propio rumen de los mismos (entre otros, Evershed et al. 2002; Martínez Marín et al. 2010). En el caso de las muestras LG3 253 y LG3 298 se identificó ácido 9-octadecenoico (C18:1), dando cuenta de la posibilidad de preservación en la matriz de materiales líticos de ácidos insaturados, los cuales se caracterizan por estar formados por enlaces dobles. Estos tipos de ácidos grasos poseen un punto de fusión más bajo que los saturados, por lo que la presencia de dobles enlaces en la estructura incide en su preservación. Asimismo, cabe destacar la forman a partir del proceso de autooxidación de ácidos grasos insaturados (Malainey et al. 1999b; Evershed 2008). Esto permite inferir la acción de diversos procesos posdepositacionales sobre las muestras analizadas. Finalmente, el análisis de los sedimentos permite descartar a priori la contaminación de los artefactos arqueológicos por migración de sustancias, dado que los ácidos grasos identificados -C16:0, C18:0 y C18:1- se presentan en forma de trazas. Su presencia podría relacionarse con en los artefactos LG3 253 (8,13\%) y LG3 313 (9,71\%) presencia de diácidos -subérico y sebácico-, los cuales se

la descomposición de materia orgánica que, a partir de procesos pedogénicos, pasa a formar parte de la matriz sedimentaria (Steele 2013).

Los resultados obtenidos permiten inferir el contacto de los artefactos arqueológicos con especies de origen animal en forma predominante, aunque es necesario profundizar los análisis a fin de relacionarlos con aquellas disponibles en el área de estudio. Si bien no existen indicios respecto de la presencia de lípidos que pueden encontrarse en especies de origen vegetal, no debe descartarse la posibilidad del contacto con aquellas.

La detección de sustancias grasas en los artefactos analizados destaca el valor de la aplicación de enfoques arqueométricos para responder preguntas arqueológicas, especialmente en ambientes donde restos de ciertos elementos (por ej.: vegetales) no han perdurado. En el caso específico de esta investigación, la utilización de una técnica de caracterización química permitió generar información complementaria para el área. Sin embargo, es menester aclarar que se trata de un proceso lento y complejo, donde diversas variables deben ser consideradas. La preservación de los compuestos constituye una necesidad fundamental para el desarrollo de este tipo de estudios, por lo que resulta vital la correcta conservación y manejo de los artefactos seleccionados para análisis. Asimismo, el conocimiento acerca de la procedencia de la muestra, su asociación con otro tipo de restos, los procesos pedogénicos particulares que afectan la matriz sedimentaria del sitio y/o los agentes que puedan contaminar la muestra posibilitan, en conjunto, arribar a interpretaciones con gran contenido explicativo. Por otro lado, la exclusión de las muestras de superficie -dado el riesgo de degradación de los compuestos a partir de su exposición a ambientes más fluctuantes- genera una reducción drástica en el número de muestras plausibles de ser sometidas a este tipo de análisis. Se refuerza así la necesidad de tomar medidas adicionales respecto de la extracción y conservación de las muestras durante los trabajos de campo así como en el laboratorio.

\begin{tabular}{|c|c|c|c|c|c|c|c|c|c|}
\hline \multirow[b]{2}{*}{ ÁCIDO GRASO } & \multicolumn{9}{|c|}{ MUESTRA } \\
\hline & $\begin{array}{r}\text { LG3 } \\
253\end{array}$ & $\begin{array}{l}\text { LG3 } \\
298\end{array}$ & $\begin{array}{l}\text { LG3 } \\
299\end{array}$ & $\begin{array}{r}\text { LG3 } \\
313\end{array}$ & $\begin{array}{c}\text { Exp. } \\
2\end{array}$ & $\begin{array}{c}\text { Exp. } \\
4\end{array}$ & $\begin{array}{c}\text { Sed. } \\
1\end{array}$ & $\begin{array}{c}\text { Sed. } \\
2\end{array}$ & $\begin{array}{c}\text { Sed. } \\
3\end{array}$ \\
\hline Subérico (ácido octanodioico) & $1.42 \%$ & - & - & $1.63 \%$ & - & - & $x$ & - & - \\
\hline Azelaico (ácido nonanodioco) & $2.0 \%$ & - & - & $11.18 \%$ & $0.13 \%$ & - & - & - & - \\
\hline Sebácico (ácido decanodioico) & - & - & - & $0.68 \%$ & - & - & - & - & - \\
\hline C14:0 (ácido tetradecanoico ) & $8.13 \%$ & - & - & $9.71 \%$ & $1.05 \%$ & $2.1 \%$ & - & - & - \\
\hline C15:0 (ácido pentadecanoico)* & $10.65 \%$ & $15.42 \%$ & - & $6.48 \%$ & $0.27 \%$ & TR & - & - & - \\
\hline C16:0 (ácido hexadecanoico) & $44.83 \%$ & $64.57 \%$ & TR & $50.17 \%$ & $25.37 \%$ & $29.35 \%$ & $x$ & TR & TR \\
\hline C16:1 (ácido 9-hexadecenoico ) & - & - & TR & - & $1.91 \%$ & $2.60 \%$ & - & - & - \\
\hline C17:0 (ácido heptadecanoico) & $9.19 \%$ & - & - & TR & $0.27 \%$ & $0.83 \%$ & - & - & - \\
\hline C18:0 (ácido octadecanoico) & $18.01 \%$ & $6.29 \%$ & - & $20.15 \%$ & $10.91 \%$ & $14.86 \%$ & $\mathrm{x}$ & TR & TR \\
\hline C18:1 (ácido 9-octadecenoico) & $3.97 \%$ & $13.72 \%$ & - & TR & $48.86 \%$ & $48.17 \%$ & TR & TR & TR \\
\hline C18:2 (ácido 9,12-octadecadienoico) & - & - & - & - & $9.95 \%$ & $1.76 \%$ & - & - & - \\
\hline $\begin{array}{c}\text { C18:3 (ácido 9,12,15- } \\
\text { octadecatrienoico) }\end{array}$ & - & - & - & - & $0.32 \%$ & TR & - & - & - \\
\hline C20:0 (ácido eicosanoico) & - & - & - & - & TR & - & - & - & - \\
\hline C20:1 (ácido 11, eicosenoico) & - & - & - & - & $0.7 \%$ & - & - & - & - \\
\hline C20:2 (ácido 11,14 eicosadienoico) & - & - & - & - & $0.2 \%$ & - & - & - & - \\
\hline $\mathrm{NI}=$ no identificado & $1.8 \%$ & $0 \%$ & $0 \%$ & $0 \%$ & $0.06 \%$ & $0.33 \%$ & $0 \%$ & $0 \%$ & $0 \%$ \\
\hline
\end{tabular}

Tabla 1. Abundancia relativa (\%) de ésteres metílicos de ácidos grasos (FAME) obtenidos por CG-FID y confirmados por CG-EM. * Lineal y ramificado. $\mathrm{TR}=$ trazas.

Table 1. Relative abundance of methyl esters of fatty acids (FAME) obtained bye CG-FID and confirmed by CG-EM. * Linear and branching. 
Vale destacar que si bien se trata de una muestra pequeña, consideramos que los resultados obtenidos constituyen un inicio alentador respecto de los objetivos planteados. En una siguiente etapa de trabajo se plantea el estudio del perfil lipídico de una mayor cantidad de artefactos líticos mediante CG-FID, CG-EM u otro tipo de técnica que se pueda aplicar para el estudio de los mismos. Asimismo, se realizarán estudios experimentales sobre muestras de origen vegetal con el objetivo de obtener información en relación con el aprovechamiento de este tipo de recurso. Por otro lado, y a pesar de la existencia de numerosos trabajos (entre otros, González de Bonaveri y Frère 2004; Bonomo et al. 2012; Flegenheimer et al. 2013; Frère et al. 2010; Illescas et al. 2012; Mazzia et al. 2016) que profundizan en el análisis de ácidos grasos recuperados en diversos soportes (alfarería, instrumentos de molienda), no existe una base de datos comparativa que permita asociar de manera segura los valores obtenidos con recursos vegetales o animales presentes en el área bajo estudio. Por consiguiente, la construcción de una base de datos del perfil de ácidos grasos presentes en la flora y fauna del Macizo es otro de los desafíos planteados a futuro.

\section{Agradecimientos}

El presente trabajo forma parte de los Proyectos PIP 0447 y PICT 2015-2038 dirigidos por la Dra. Nora V. Franco, a quien agradezco su aporte respecto del análisis del material lítico. A la Dra. Natalia Mazzia por su colaboración respecto de la interpretación de los resultados obtenidos. A Lucas, Brenda, Vicky y Clara por la colaboración prestada en la elaboración y realización de la experimentación. A las empresas Minera Piedragrande S.A.M.I.C.A. Y F. y Minera Triton Argentina S.A por facilitar el acceso a los sitios. A la Lic. Nerina Pisani por su inmensa generosidad. Finalmente, a los revisores designados, cuyos valiosos aportes incrementaron la calidad del presente artículo.

Buenos Aires, noviembre de 2019

\section{Bibliografía}

Aguerre, A.M. (2003). La Martita: Ocupaciones de 8000 años en la Cueva 4. En A. Aguerre (Ed.) Arqueología y Paleoambiente en la Patagonia Santacruceña Argentina (29-61). Ediciones del autor. Buenos Aires.

Aschero, C.A. (1975). Ensayo para una clasificación morfológica de artefactos líticos aplicada a estudios tipológicos comparativos. Informe al CONICET. MS.

Aschero, C.A. (1983). Ensayo para una clasificación morfológica de artefactos líticos aplicada a estudios tipológicos comparativos. Informe al CONICET. MS.

Babot, M.P., N. Mazzia y C. Bayón. (2007). Procesamiento de recursos en la región pampeana bonaerense: aportes del instrumental de molienda de las localidades arqueológicas El Guanaco y Cerro La China. En C. Bayón, A. Pupio, M.I. González, N. Flegenheimer y M. Frère (Eds.) Arqueología en las pampas (635-660). SAA. Bahía Blanca.

Bonomo, M., M. Colobig y N. Mazzia. (2012). Análisis de residuos orgánicos y microfósiles silíceos de la "cuchara" de cerámica del sitio arqueológico Cerro Tapera Vázquez (Parque Nacional Pre-Delta, Argentina). En R. Museu Arq. Etn. 22 (31-50). São Paulo.

Borrero, L.A, J. Charlín, R. Barberena, F.M. Martín, K. Borrazzo y L. L' Heureux. (1998). Circulación humana y modos de interacción al sur del Río Santa Cruz. En L.A. Borrero y N.V. Franco (Coords.) Arqueología del extremo sur del continente americano. Resultados de nuevos proyectos. $1^{\circ} \mathrm{Ed}$. - CONICET.

Brook, G.A., N.V. Franco, P. Ambrústolo, M.V. Mancini, L. Wang, P.M. Fernández. (2015). Evidence of the earliest humans in the Southern Deseado Massif (Patagonia, Argentina), Mylodontae, and changes in water availability. En Quaternary International 363 (107-125).

Buonasera, T. (2005). Fatty acid analysis of prehistoric burned rocks: a case study from central California. En Journal of Archaeological Science 32 (957-65). Elsivier.

Buonasera, T. (2007). Investigating the presence of ancient absorbed organic residues in groundstone using GCeMS and other analytical techniques: a residue study of several prehistoric milling tools from central California. En Journal of Archaeological Science 34 (1379-90). Elsivier.

Chaile, C., I. Lantos, M. Maier, G. Cassiodoro y A. Tessone. (2018). Análisis de residuos orgánicos en tecnología cerámica durante el Holoceno tardío en el centro-oeste de Santa Cruz, Argentina. En Intersecciones en Antropología 19 (133-143). Facultad de Ciencias Sociales, UNCPBA, Argentina.

Cordero, J.A. y R.J. March. (2013). Análisis de ácidos grasos en fragmentos cerámicos del Nordeste de la Patagonia argentina por CG y CG-MS. En M. Ramos, M. I.anza, V. Helfer, V. Pernicone, F. Bognanni, C. Landa, V. Aldazábal y M. Fernández (Eds.) Arqueometría Argentina: Estudios pluridisciplinarios. Programa de Arqueología y Estudios Pluridisciplinarios, Departamento de Ciencias Sociales, Universidad Nacional de Luján.

Durán, V., A. Gil, G. Neme y A. Gasco. (2003). El Verano: ocupaciones de 8900 años en la Cueva 1 (Santa Cruz, Argentina). En A. Aguerre (Ed.) Arqueología y Paleoambiente en la Patagonia Santacruceña Argentina (93-120). Ediciones del Autor, Buenos Aires.

Evershed, R.P., C. Heron, and L.J. Goad. (1990). Analysis of organic residues of archaeological origin by high temperature gas chromatography/mass spectrometry. En Analyst 115 (1339-42). 
Evershed, R.P., S.N. Dudd, M.S. Copley, R. Berstan, A.W. Stott, H. Mottram, S.A. Buckley y Z. Crossman. (2002). Chemistry of Archaeological Animal Fats. En Accounts of Chemical Research Vol. 35 (8), 660-668. American Chemical Society.

Evershed, R.P. (2008). Organic Residue Analysis Archeology: The Archaeological Biomarker Revolution. En Archaeometry 50 (6), 895-924. University of Oxford.

Franco, N.V., P. Ambrústulo, A. Acevedo, N. Cirigliano y M. Vommaro. (2013). Prospecciones en el sur del Macizo del Deseado (Provincia de Santa Cruz, Argentina). Los casos de La Gruta y Viuda Quenzana. En A.F. Zangrando, R. Barberena, A. Gil, G. Neme, M. Giardina, L. Luna, C. Otaola, S. Paulides, L. Salgán y A. Tívoli (Eds.) Tendencias teórico-metodológicas y casos de estudio en la arqueología de la Patagonia (371-378). Museo de Historia Natural de San Rafael, Altuna Impresores, Buenos Aires.

Flegenheimer, N., N. Mazzia y M.P. Babot. (2013). Estudios de detalle sobre una piedra discoidal pampeana. En Intersecciones en Antropología 14 (499-505). Facultad de Ciencias Sociales, UNCPBA, Argentina.

Frère, M.M., M.I. González, D. Constenla y C. Bayón. (2010). Experimentación con recursos actuales mediante el empleo de análisis químicos. En M. Berón, L. Luna, M. Bonomo, C. Montalvo, C. Aranda y M. Carrera Aizpitarte (Eds.) Mamül Mapu: pasado y presente desde la arqueología pampeana (65-76). Editorial Libros del Espinillo, Ayacucho.

Frére, M.M., M.I. González, D. Chan y M. Flores. (2016). Evidencias químicas de preparación de alimentos en la alfarería de la Depresión del rio Salado. En Anuario de Arqueología 8 (141-151). Rosario.

Gómez Otero, J., D. Constenla y V. Schuster. (2014). Análisis de isotopos estables de carbón y nitrógeno y cromatografía gaseosa en cerámica arqueológica del nordeste de la provincia de Chubut (Patagonia Argentina). En Arqueología 20 (2), 263-284. Instituto de Arqueología, FFyL, UBA.

González de Bonaveri, M.I. y M.M. Frère. (2004). Analysis of Potsherd Residues and Vessel Use in Hunter-Gatherer-Fisher Groups (Pampean Region, Argentina). General Sessions and Posters, University of Liège, Belgium. En BAR International series 1270 (Ed.) Archaeometry (27-36). Oxford.

Illescas, F., A. Cañizo, M.G. Musaubach y M.A. Berón. (2012). De ollas, aceites y otras yerbas. Análisis complementarios sobre alfarería pampeana. En M.P. Babot, M. Marschoff y F. Pazzarelli (Eds.) Las manos en la masa: arqueologías, antropologías e historias de la alimentación en Suramérica (387-404). Facultad de Filosofía y Humanidades, Museo de Antropología (UNC), Instituto Superior de Estudios Sociales (UNT), Universidad Nacional de Córdoba.
Lantos, I.J. (2013). De residuos y vasijas: prácticas alimentarias en el oeste tinogasteño. Delineando prácticas de la gente del pasado: Los procesos sociohistóricos del oeste catamarqueño: 379 - 402. Publicaciones de la Sociedad Argentina de Antropología. Buenos Aires.

Malainey, M.E., R. Przybylski y B.L. Sheriff. (1999a). The Fatty Acid Composition of Native Food Plants and Animals of Western Canada. En Journal of Archaeological Science 26 (83-94).

Malainey, M.E., R. Przybylski y B.L. Sheriff. (1999b). The Effects of Thermal and Oxidative Degradation of Food Plants and Animals of Western Canada: Implications for the Identification of Archaeological Vessel Residues. En Journal of Archaeological Science 26 (95-103).

Mancini, M.V., N.V. Franco y G.A. Brook. (2013). Paleoenviromental and early human occupation of saouthernmost South America (South Patagonia, Argentina). En Quaternary International 299 (13-22). Elsevier.

Martínez Marín, A., M. Hernández Pérez, L. Pérez Alba, G. Gómez Castro y D. Carrión Pardo. (2010). Metabolismo de los lípidos en los rumiantes. Revista Electrónica de Veterinaria 11 (1695-7504).

Mazzia, N. (2010). Lugares y paisajes de cazadores recolectores en la pampa bonaerense: cambios $y$ continuidades durante el Pleistoceno final - Holoceno. Tesis de doctorado. Universidad Nacional de La Plata, Facultad de Ciencias Naturales y Museo.

Mazzia, N., C. Weitzel y H. De Angelis. (2016). De usos y recursos. Análisis funcional y de sustancias grasas mediante cromatografía gaseosas sobre artefactos líticos tallados. En Revista del Museo de Antropología 9 (2), 37-50. Facultad de Filosofía y Humanidades, Universidad Nacional de Córdoba, Argentina.

Miotti, L. y M. Salemme. (2004). Poblamiento, movilidad y territorios entre las sociedades cazadoras-recolectoras de Patagonia. En Complutum 15 (177-206).

Panza, J.L. y G. Marin. (1998). Geología. Hoja Geológica 4969-I "Gobernador Gregores", Provincia de Santa Cruz. En Boletín 239 (1-104). SEGEMAR, Buenos Aires.

Steele, V. (2013). Organic Residues in Archaeology: The Highs and Lows of Recent Research. En Archaeological Chemistry VIII (89-108). American Chemical Society, Washington, D.C.

Stoessel, L., G. Martínez y D. Constenla. (2015). Análisis preliminar de ácidos grasos recuperados de cerámicas arqueológicas del curso inferior del rio Colorado (Norpatagonia oriental): aportes para la subsistencia de grupos cazadores recolectores. En Magallania Vol. 43 (1), 231-249. Chile. 